\title{
Giant mediastinal liposarcoma resected by median sternotomy: a case report
}

\author{
Huijun Zhang ${ }^{1}$, Nadier Yimin ${ }^{1}$, Zelai $\mathrm{He}^{2}$, Xiaofeng Chen ${ }^{1}$ \\ ${ }^{1}$ Department of Cardiothoracic Surgery, Huashan Hospital of Fudan University, Shanghai, China; ${ }^{2}$ Department of Radiation Oncology, The First \\ Affiliated Hospital of Bengbu Medical College, Bengbu, China \\ Correspondence to: Xiaofeng Chen. Department of Cardiothoracic Surgery, Huashan Hospital of Fudan University, Shanghai, China. \\ Email: zhj6746@163.com; Zelai He. Department of Radiation Oncology, The First Affiliated Hospital of Bengbu Medical College, Bengbu, China. \\ Email: he.ze.lai@163.com.
}

\begin{abstract}
Liposarcoma is a type of common tumor in soft tissue, but liposarcoma originating in the mediastinum is rare. Here, we report a case of the anterior mediastinal liposarcoma resected by a median sternal incision and complicated with reexpansion pulmonary edema after surgery. A 68-year-old female patient with chest tightness and shortness of breath for more than 2 years, recently presents with increased chest tightness and shortness of breath, as well as right upper extremity and lower back pain. Enhanced chest CT scan showed an uneven and low-density mass in the anterior mediastinum with clear border. Most of the mass showed fat density, the anterior part of the mass was solid, and the liquid density was seen in the pericardial cavity. Surgery was performed with a median sternal incision, and part of the pericardium and the innominate vein wall were removed during the removal of the entire liposarcoma. The size of the tumor was about $20 \mathrm{~cm} \times 10 \mathrm{~cm} \times 8 \mathrm{~cm}$. The patient developed a reexpansion pulmonary edema after the giant mediastinal liposarcoma resection, but she was discharged successfully on the 10th postoperative day with the treatment by anti-glucocorticoids and diuretics. Postoperative pathology showed welldifferentiated liposarcoma. Now within the half-year follow-up, the patient remained well and there is no sign of recurrence. Median sternotomy is considered to be a good surgical procedure for giant mediastinal liposarcomas. Attention should be given to prevent reexpansion pulmonary edema after surgery.
\end{abstract}

Keywords: Mediastinal liposarcoma; median sternotomy; reexpansion pulmonary edema

Submitted Mar 01, 2020. Accepted for publication Sep 02, 2020.

doi: $10.21037 /$ tcr-20-1270

View this article at: http://dx.doi.org/10.21037/tcr-20-1270

\section{Introduction}

Liposarcoma arising from the mediastinum is uncommon (1). Mediastinal liposarcoma is not sensitive to radiotherapy and chemotherapy, hence complete resection is required (2). With the rapid growth and large volume of the tumor, reexpansion pulmonary edema is prone to occur after the tumor removal, but there are few reports about reexpansion pulmonary edema after mediastinal liposarcoma resection. We here present a patient who underwent resection of the giant anterior mediastinal liposarcoma via a median sternal incision and postoperative reexpansion pulmonary edema. We present the following case in accordance with the CARE reporting checklist (available at http://dx.doi. org/10.21037/tcr-20-1270).

\section{Case presentation}

A 68-year-old woman was admitted to our department on September 20, 2019, with complaints of chest tightness and shortness of breath for more than 2 years, with chest tightness and shortness of breath being worse than before. The right upper limb and lower back were also in pain, but the patient was in good mental health. The patient had no history of fever, cough, hemoptysis, significant weight loss, or other symptoms. This patient has a 20 years of 

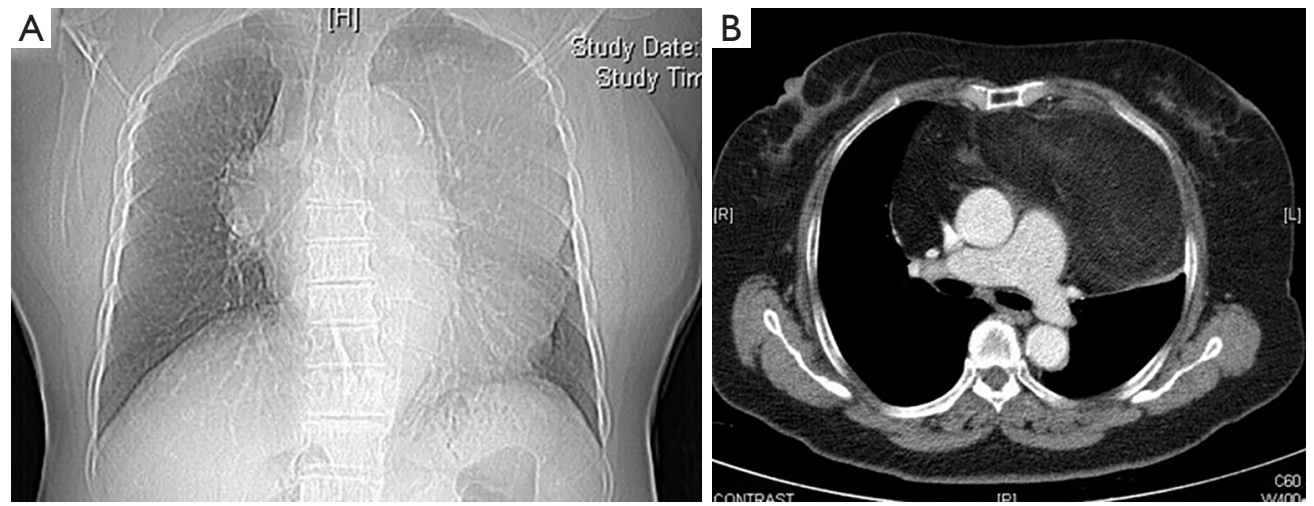

Figure 1 A huge mediastinal tumor in A 68-year-old woman. Chest X-ray showing a huge mediastinal tumor at the first visit (A); enhanced CT showed a large mass of $20 \mathrm{~cm} \times 10 \mathrm{~cm} \times 8 \mathrm{~cm}$ in size with low density in the anterior mediastinum extending anteriorly into the left hemithorax (B).

history of hypertension with blood pressure up to $180 / 110$ $\mathrm{mmHg}$, but her blood pressure was already under control. Other past medical history includes obsessive-compulsive disorder and cerebral infarction for 15 years. The patient's personal and family medical history was unremarkable. The physical examination showed the trachea being slightly to the right, no chest deformity, normal respiratory motility, solid sound on percussion of left lung, and clear lung breathing at the auscultation of the right lung field. Her laboratory findings showed no significant abnormality. The chest $\mathrm{X}$-ray result demonstrated a giant mediastinal tumor (Figure 1A). An enhanced chest computed tomography (CT) scan revealed a large mass of $20 \mathrm{~cm} \times 10 \mathrm{~cm} \times 8 \mathrm{~cm}$ in size in the anterior mediastinum, invading the pericardium and extending into the bilateral thoracic cavity and causing a mediastinal shift and lung collapse. Most of the mass showed fat density on the CT scan, the CT value was about $-56 \mathrm{HU}$, and the anterior part of the mass was solid while the CT value was about 20 HU. A small amount of liquid was seen in the pericardial cavity, as shown in the figure (Figure $1 B$ ). The chest radiology specialists believed that the mass shown in the CT scan might be a mediastinal liposarcoma.

Therefore, surgical resection was performed on September 28, 2019. Median sternotomy was chosen to allow complete access to the bilateral thoracic cavity for a large tumor resection with the patient under general anesthesia (Figure 2A). The tumor was located in the anterior mediastinum with most part of it being in the left thoracic cavity, partially invading the pericardium and superior vena cava. During the operation, part of the pericardium was resected, and then part of the innominate vein was also resected with a vascular staple, and finally the tumors were completely separated and dissected (Figure 2B). The operation time was $98 \mathrm{~min}$, the intraoperative blood loss was about $700 \mathrm{~mL}$, and no blood transfusion was performed. The patient was transferred to the ICU with tracheal intubation.

The patient failed to show signs for removal of tracheal intubation, and still required assisted breathing through a ventilator on September 30, 2019. A bedside chest $\mathrm{X}$-ray showed a decrease in lung field transillumination and an increase in lung texture (Figure 3). Then antiglucocorticoids and diuretic treatment were given for three consecutive days. The tracheal intubation was successfully removed on October 5, 2019. Postoperative recovery of the patient was good, and she was successfully discharged on October 7, 2019. Postoperative pathology showed well-differentiated liposarcoma and incomplete capsule. Immunohistochemical results are as follows: CK (-), VIM $(+)$, Ki67 (3\% +), SMA (-), CD34 (-), CD68 (-), S100 (+), CDK4 (+), MDM2 (-), P16 (+). The patient remained well and showed no sign of recurrence on March 10, 2020 (Figure 4). The timeline of the symptoms, treatment, and follow-up of this case is listed in Figure 5. All procedures performed in studies involving human participants were in accordance with the ethical standards of the institutional research committee and with the Helsinki Declaration (as revised in 2013). Written consent was obtained from the patient for publication of this case report. 

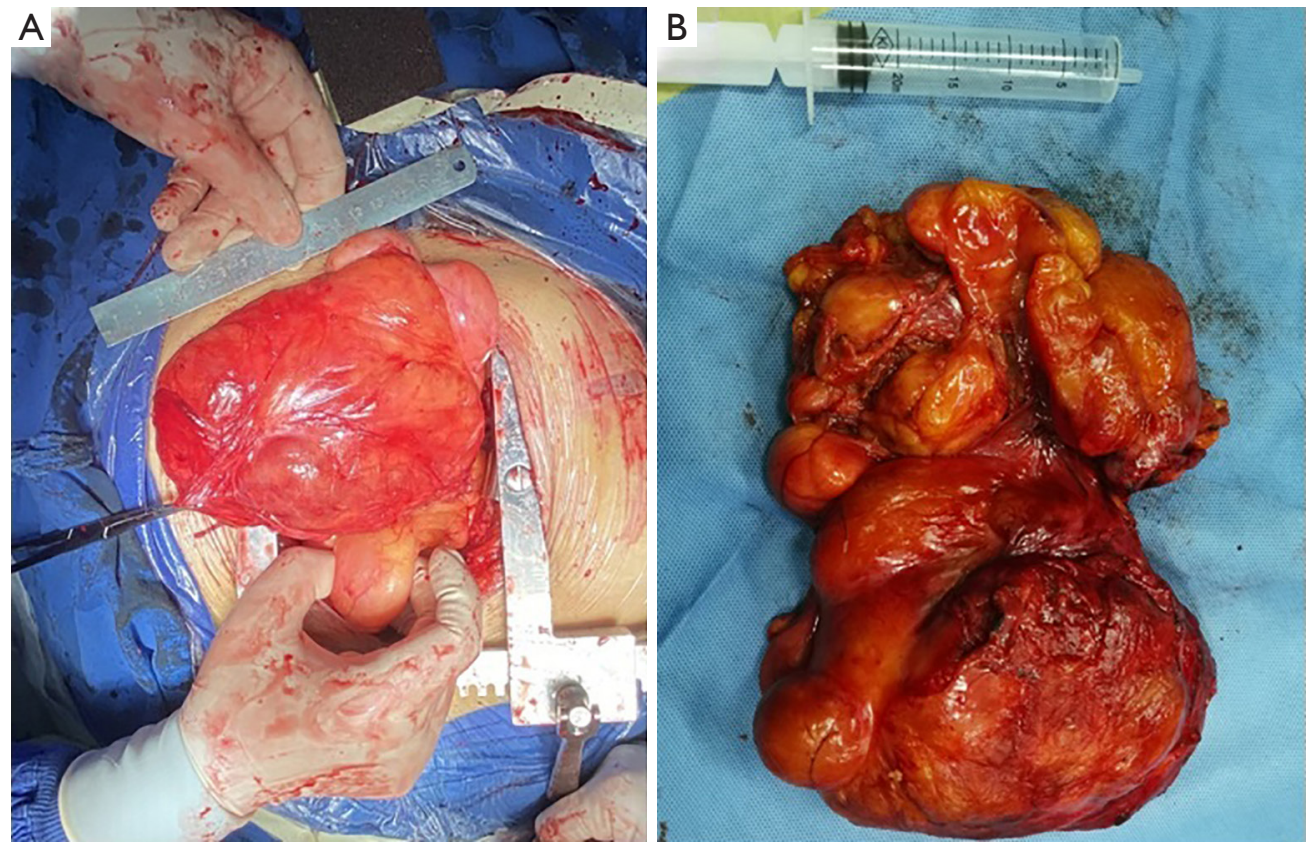

Figure 2 The resection of mediastinal liposarcoma. A good operative field was obtained by median sternotomy (A); the tumors were approximately $20 \mathrm{~cm} \times 10 \mathrm{~cm} \times 8 \mathrm{~cm}$ in size after complete resection (B).

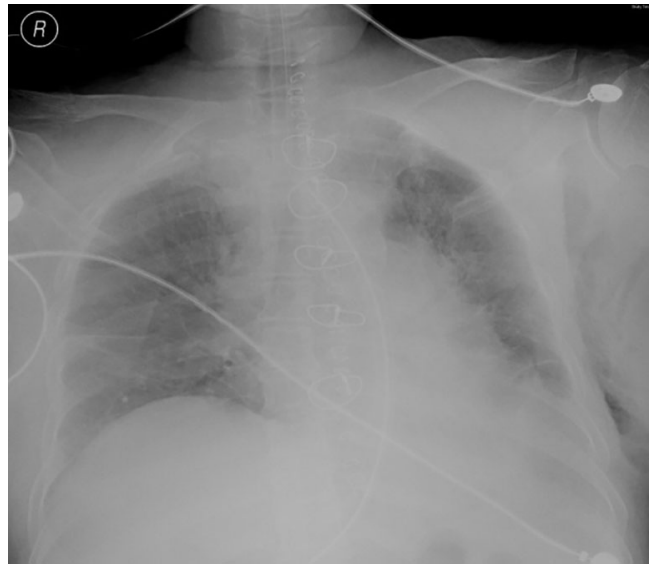

Figure 3 The chest X-ray showed lung field transillumination decreased, lung texture increased, lung field blur in the second day after surgery.

\section{Discussion}

Liposarcoma is a common tumor in soft tissue, but liposarcoma originating in the mediastinum is rare (3). Liposarcoma can occur anywhere in the mediastinum. However, it is more common in the lower part of the anterior mediastinum, often biased to one side of the chest,

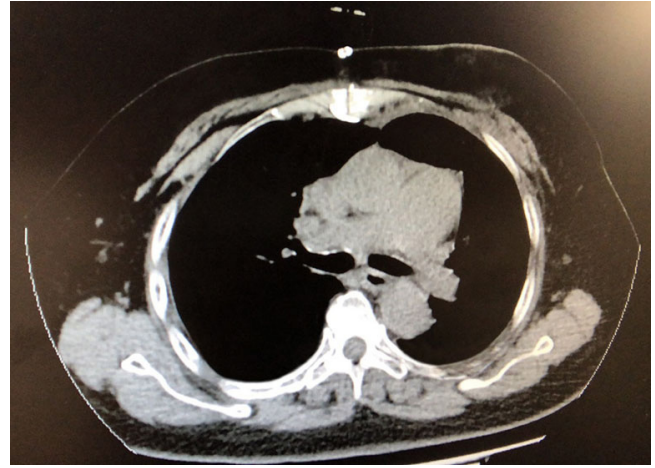

Figure 4 Within the half-year follow-up, the CT scan of the patient showed no sign of recurrence.

and usually relatively large when found. When the huge mass is removed, it is easy to cause reexpansion pulmonary edema after surgery, but there are few reports in the literature. Therefore, it is important for us to raise awareness of this rare disease.

Common symptoms of mediastinal liposarcoma include coughing, difficulty breathing, wheezing, chest pain and weight loss. In this case, the mass was huge, and located in the middle and lower part of the anterior mediastinum, biased toward the left chest cavity, the patient presented 


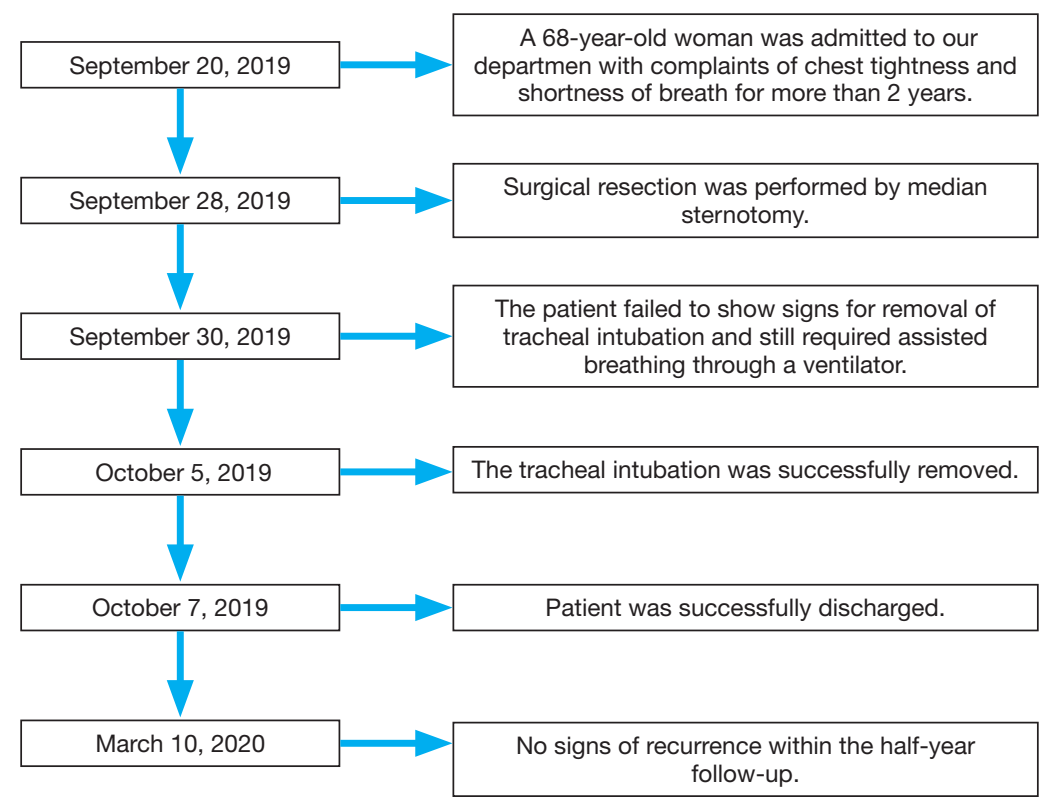

Figure 5 The timeline of the symptoms, treatment, and follow-up of this patient.

with symptoms of chest tightness and shortness of breath. However, there have been cases of giant mediastinal liposarcoma with no physical symptoms (4). The appearance of these symptoms may be related to the degree of compression of important organs. The morphology of mediastinal liposarcoma is often more irregular than other tumors, and it tends to grow towards areas with weak resistance. Generally, the liposarcoma is widely distributed and grows rapidly in the mediastinum. In this case, liposarcoma invaded part of the pericardium and superior vena cava, which also made the surgery difficult and affected the patient's prognosis.

The CT scan of mediastinal liposarcoma showed the invasive growth into the surrounding tissues and its CT value generally range from -80 to $40 \mathrm{HU}$. The lower the CT value of mediastinal liposarcoma, the higher the degree of differentiation. In this case, the CT value of mediastinal liposarcoma is about $-56 \mathrm{HU}$, and the CT value of the portion of tumor tissue can reach up to $20 \mathrm{HU}$. The pathology diagnosis of this case was well differentiated liposarcoma, and the results of immunohistochemistry were as follows: CDK4 (+), MDM2 (-), P16 (+). Immunohistochemical detection of MDM2, CDK4 and 16 in tumors provides a useful diagnostic tool for distinguishing well differentiated liposarcoma from other adipocytic tumors. p16 is the most sensitive and specific marker molecule, and the combination of CDK4 and p16 has even more diagnostic value $(5,6)$. The etiology of liposarcoma is unclear and may be related to the following factors: trauma, hematoma, viral infection and radiation therapy.

The liposarcoma is not sensitive to radiotherapy and chemotherapy. The best treatment is early radical resection. If the resection is incomplete, it is common to relapse after surgery. Recurrence is associated with incomplete surgical resection and the amount of tumor tissue left after surgery. Many surgical methods can be considered. Clamshell incisions are used to resect bilateral pulmonary metastases and large mediastinal tumors (7). However, in this case, the tumor reaches the top of the chest and down to the top of the diaphragm. Therefore, Clamshell incisions are difficult to achieve sufficient exposure in this case. It has been reported that mediastinal liposarcoma was removed through a left thoracic incision (8), but the tumor invaded the superior vena cava and pericardium in this case, so this type of incision is not suitable for fully exposing the superior vena cava and pericardium. Median sternotomy is a common approach to resecting mediastinal tumors. In this case, we chose a standard median sternotomy with an open field of view that fully exposed the tumors on both sides of the thoracic cavity and removed the affected part of the pericardium and superior vena cava wall. This surgery achieved the basic standards required of a complete resection. In 
this case, huge mediastinal liposarcoma causes longterm compression of the lungs, and the lungs are rapidly decompressed after the mass is removed, resulting in postoperative reexpansion pulmonary edema. After treatment with glucocorticoids and diuresis, the patient was successfully discharged. Liposarcoma is characterized by its large volume and histological subtypes, which are closely related to clinical symptoms and prognosis. In 2013, WHO classified liposarcoma into four subtypes: well-differentiated, dedifferentiated, myxoid/round cell, and pleomorphic $(9,10)$. Among them, dedifferentiated and pleomorphic liposarcoma have a poor prognosis. This patient with highly differentiated histological subtype may have a relatively good prognosis.

In addition, molecular targeted therapy of liposarcoma has also been extensively studied, and the research results are remarkable, which will be good news for patients with liposarcoma $(11,12)$.

In our case, a standard median sternotomy was needed for a better exposure to completely remove the tumor, including the affected part of the superior vena cava and pericardium. However, some studies have reported that the 5 -year recurrence rate of mediastinal liposarcoma can reach $50 \%(7,13)$. The follow-up time of this case is still limited, and the condition of the patient remains to be observed.

\section{Conclusions}

Median sternotomy is considered as a good surgical procedure for giant mediastinal liposarcomas. Since huge mediastinal liposarcoma causes long-term compression of the lungs, attention should be given to prevent reexpansion pulmonary edema after surgery.

\section{Acknowledgments}

Funding: None.

\section{Footnote}

Reporting Checklist: The authors have completed the CARE reporting checklist. Available at http://dx.doi.org/10.21037/ tcr-20-1270

Conflicts of Interest: All authors have completed the ICMJE uniform disclosure form (available at http://dx.doi. org/10.21037/tcr-20-1270). The authors have no conflicts of interest to declare.
Ethical Statement: The authors are accountable for all aspects of the work in ensuring that questions related to the accuracy or integrity of any part of the work are appropriately investigated and resolved. All procedures performed in studies involving human participants were in accordance with the ethical standards of the institutional research committee and with the Helsinki Declaration (as revised in 2013). Written consent was obtained from the patient for publication of this case report. The patient gave consent for his clinical details as well as any identifying images to be published in this study.

Open Access Statement: This is an Open Access article distributed in accordance with the Creative Commons Attribution-NonCommercial-NoDerivs 4.0 International License (CC BY-NC-ND 4.0), which permits the noncommercial replication and distribution of the article with the strict proviso that no changes or edits are made and the original work is properly cited (including links to both the formal publication through the relevant DOI and the license). See: https://creativecommons.org/licenses/by-nc-nd/4.0/.

\section{References}

1. Krishnasamy S, Krishna Nair A, Hashim SA, et al. Mediastinal liposarcoma: a rare visceral mediastinal tumour. Interact Cardiovasc Thorac Surg 2019;29:976-7.

2. Hahn HP, Fletcher CD. Primary mediastinal liposarcoma: clinicopathologic analysis of 24 cases. Am J Surg Pathol 2007;31:1868-74.

3. Politis J, Funahashi A, Gehlsen JA, et al. Intrathoracic lipomas. Report of three cases and review of the literature with emphasis on endobronchial lipoma. J Thorac Cardiovasc Surg 1979;77:550-6.

4. Mani VR, Ofikwu G, Safavi A. Surgical resection of a giant primary liposarcoma of the anterior mediastinum. J Surg Case Rep 2015;2015:rjv126.

5. Thway K, Flora R, Shah C, et al. Diagnostic utility of p16, CDK4, and MDM2 as an immunohistochemical panel in distinguishing well-differentiated and dedifferentiated liposarcomas from other adipocytic tumors. Am J Surg Pathol 2012;36:462-9.

6. Thway K. Well-differentiated liposarcoma and dedifferentiated liposarcoma: An updated review. Semin Diagn Pathol 2019;36:112-21.

7. Toda M, Izumi N, Tsukioka T, et al. Huge mediastinal liposarcoma resected by clamshell thoracotomy: a case report. Surg Case Rep 2017;3:16. 
8. Zhang M, Zhang S, Shi H, et al. Resection of a huge mediastinal well-differentiated liposarcoma involving left thoracic cavity. J Cardiothorac Surg 2019;14:148.

9. Jo VY, Doyle LA. Refinements in Sarcoma Classification in the Current 2013 World Health Organization Classification of Tumours of Soft Tissue and Bone. Surg Oncol Clin N Am 2016;25:621-43.

10. Jo VY, Fletcher CD. WHO classification of soft tissue tumours: an update based on the 2013 (4th) edition. Pathology 2014;46:95-104.

Cite this article as: Zhang H, Yimin N, He Z, Chen X. Giant mediastinal liposarcoma resected by median sternotomy: a case report. Transl Cancer Res 2020;9(10):6522-6527. doi: 10.21037/ tcr-20-1270
11. Patel RB, Li T, Liao Z, et al. Recent translational research into targeted therapy for liposarcoma. Stem Cell Investig 2017;4:21.

12. Crago AM, Dickson MA. Liposarcoma: Multimodality Management and Future Targeted Therapies. Surg Oncol Clin N Am 2016;25:761-73.

13. Park JO, Qin LX, Prete FP, et al. Predicting outcome by growth rate of locally recurrent retroperitoneal liposarcoma: "the one centimeter per month rule". Ann Surg 2009;250:977-82. 